\title{
Tables of cases and legislation
}

\section{TABLE OF CASES}

\section{Canada}

Air Canada Pilots Association v ACE [2007] OJ No 89 (QL), 2007 CanLII 337 (Ont Sup

Ct) 138

BCE Inc v 1976 Debenture holders 2008 SCC 69, [2008] 3 SCR 560 115, 116, 137, 138

Peoples Department Store Inc (Trustee of) v Wise 2004 SCC 68, [2004] 3 SCR $461 \quad 9,11,64$, 115, 116, 137-8

\section{Taiwan}

Procomp Informatics case, 2004215

\section{United Kingdom}

Allen v Gold Reefs of West Africa Ltd [1900] 1 Ch $656 \mathbf{2 3 1}$

Burnes v Pennell [1849] 2 HL Cas 49752

Case of Sutton's Hospital (1612) 10 Coke Rep 22b, 77 ER $960 \quad 50$

Foss v Harbottle (1843) 67 ER 189231

Kinder v Taylor [1825] 3 LJ Ch 68 52, 56

Salomon v Salomon \& Co Ltd [1897] AC $22 \quad 231$

Trevor v Whitworth (1887) 12 App Cas 409231

\section{United States of America}

American Int'l Group Inc, in re 965 A 2d 763 (Del Ch 2009) 186

Aronson v Lewis 473 A 2d 805

(Del Sup Ct 1984) 181, 183, 188, 259, 268

ATA (American Trucking Associations Inc) v LA (City of Los Angeles) 577 F Supp 2d 1110 (CD Cal 2008) $\mathbf{2 6 8}$

ATA (American Trucking Associations Inc) v LA (City of Los Angeles) 559 F 3d 1046 (9th Cir 2009) 264, 268

ATA (American Trucking Associations Inc) v LA (City of Los Angeles) 596 F 3d 602 (9th Cir 2010) 268

Atherton v FDIC 519 US 213 (1997) 178, 180, 200

Bates v Dresser 251 US 524 (1920) 200

Beam, ex rel Martha Stewart Living Omnimedia Inc v Stewart 845 A 2d 1040 (Del 2004) 182, 223

Beanal v Freeport-McMoRan 969 F Supp 362 (ED La1997) 97

Beard v Elster 60 A 2d 731 (Del Ch 1960) 201

Biondi v Scrushy 820 A 2d 1148 (Del Ch 2003) 223

Brehm v Eisner 746 A 2d 244 (Del Sup Ct 2000) 182, 183, 189, 201, 268 
Briggs v Spaulding 141 US 132 (1891) 178

Caremark Int'1 Inc, in re 698 A 2d 959 (Del Ch 1996) 183, 186, 200

Citigroup Shareholders Derivative Litigation, in re 964 A 2d 106 (Del Ch 2009) 5, 13, 163, 164-7, 174, 177, 178, 180-94, 196, 199

Citigroup Shareholders Derivative Litigation, Second Amended Complaint 197

Citizens United v Federal Election Commission 558 US $50,130 \mathrm{SCt}$ $876(2010)$ 116, 133

Credit Lyonnais Bank Nederland NV v Pathe Communications Corp [1991] WL 277613 (Del Ch) 133

Dodge v Ford Motor Co 170 NW 668 (Mich 1919) 10, 73, 101, 102, 110-12, 117, 192

Doe v Unocal 248 F 3d 915 (9th Cir 2011) 90

Erie Railroad Co v Tompkins 304 US 64 (1938) 178

FDIC v McSweeney 976 F 2d 532 (9th Cir 1992) 196, 200

Flores v So Peru Copper Corp 343 F 3d 140 (2d Cir 2003) 97

Graham v Allis-Chalmers Mfg Co 188 A 2d 125 (Del 1963) 184, 201

Guth v Loft see Loft Inc v Guth Hoye v Meek 795 F 2d 893 (10th Cir 1986) $\mathbf{1 8 6}$

Hoyt v Thompson's Executor 19 NY 207 (1859) 48

JI Case Co v Borak 377 US 426 (1964) 175

Jones v HF Ahmanson \& Co $460 \mathrm{P}$ 2d 464 (Cal 1969) 200
Joy v North 692 F 2d 880 (2d Cir 1982), cert den 460 US 1051 (1983) 174, 200

Loft Inc v Guth 2 A 2d 225 (Del Ch 1938), aff'd sub nom Guth v Loft Inc 5 A 2d 503 (Del 1939) 72

Louis K Liggett Co v Lee 288 US 517 (1933) 190

McDermott Inc v Lewis 531 A 2d 206 (Del 1987) 177, 199

Manson v Curtis 223 NY 313, 119 NE 559 (1918) 47-8

Michelson v Duncan 407 A 2d 211 (Del 1979) 188, 201

N Am Catholic Programming Found Inc v Gheewalla $930 \mathrm{~A}$ 2d 92 (Del 2007) 192

Netsmart Technologies Inc Shareholders Litigation, in re 924 A 2d 1140 (Del Ch 2007) 34

Oracle Derivative Litigation, in re $824 \mathrm{~A} 2 \mathrm{~d} 917$ (Del Ch 2003) 224

Paramount Communications v Time Inc 571 A 2d 1140 (Del 1989) 11, 74, 113, 133, 134

Paramount Communications Inc v QVC Network Inc 637 A 2d 34 (Del 1994) 72, 113

Revlon Inc v MacAndrews \& Forbes Holdings Inc $506 \mathrm{~A}$ (2d) 173 (Del 1986) 10, 11, 29, 72, 101, 102, 110, 112-14, 134

Santa Fe Indus Inc v Green 430 US 462 (1977) 201

Shlensky v Wrigley 237 NE 2d 776 (Ill App Ct 1968) 73, 109

Smith v Van Gorkom 488 A 2d 858 (Del 1985) 183, 184 
Sosa v Alvarez-Macain 542 US 692 (2004) 75

Steiner v Myerson 1995 WL 441999 (Del Ch 1995) 189

Stone v Ritter 911 A (2d) 362 (Del 2006) 183, 185, 186

Unisuper Ltd v News Corp 2005 WL 3529317 (Del Ch 2005) 200

United States v Carroll Towing Co 159 F 2d 169 (2d Cir 1947) 199

Unocal v Mesa Petroleum Co 493 A 2d 946 (Del 1985) 11, 72, 133, 134

Walt Disney Co, in re 825 A 2d 275 (Del Ch 2003), 906 A 2d 27 (Del 2006) 184, 189, 190, 191, 194, 201

WorldCom Inc Securities Litigation, in re 346 F Supp 2d 628 (SD NY 2004) 186, 187

\section{TABLE OF LEGISLATION}

Australia

Corporate Law Reform Act 1992 239, 247

Corporations Act 2001 (Cth) Pt 5.3A 238 s 198A 47 s $411 \quad 238$

Uniform Companies Act 1961 (Cth) $\mathbf{6 3}$

Australian Stock Exchange Corporate Governance

Principles and

Recommendations (ASX CGC 2010) 282, 288

Pr 3282
Australian Stock Exchange (ASX) Listing Rules, 2008 r $4.10 .3 \quad 281$

\section{Canada}

Canada Business Corporations Act (CBCA) 116, 135-8, 141

$$
\begin{array}{ll}
\text { s } 2 & \mathbf{1 3 6} \\
\text { s } 42 & \mathbf{1 1 8} \\
\text { s } 102 & \mathbf{6 4} \\
\text { s } 238 & \mathbf{1 3 6}
\end{array}
$$

\section{Malaysia}

Capital Market and Services Act 2007231

Companies Act 1965 16, 229, 230, 232-3, 234, 244, 246

Pt IV, Div 3A 231, 232 s $4 \quad 246$

s $67 \mathrm{~A} \quad 233$

s $68 \quad 233$

s 69D 233

s 69L 231

s $70 \quad 232$

s $84 \quad 232$

s $111 \quad 246$

s $115 \quad 232$

s $115(2) \quad 246$

s $118 \quad 246$

ss $132 \mathrm{~A}-\mathrm{E} \quad \mathbf{2 3 2}$

s $132 \mathrm{C} \quad 232$

s 132E 232

s $132 \mathrm{G} \quad \mathbf{2 3 2}, \mathbf{2 3 3}$

s $132 \mathrm{G}(6) \quad 233$

s $134 \quad 232$

s $141 \quad 232$

s $158 \quad 231$

s $165 \quad 232$

s 165(6) 232

s 176 236-7, 240, 242, 243, 246

s 176(10A) 233, 236 
s $365 \quad 233$

Sch 8, Pt II 246

Companies Enactment 1897230

Companies Enactment No 20, 1917230

Companies Enactment 1927 230, 231

Companies Ordinance 1940

(Straits Settlement) $\mathbf{2 3 1}$

Malaysian Companies (Amendment) Act(No2) Act $1998 \quad 246$

Pengurusan Danaharta Nasional Berhad Act 1998 (Danaharta Act) 237, 242

Securities Commission Act 1993231

Bursa Malaysia Listing Requirements $\mathbf{2 3 1}$

Malaysian Code of Corporate Governance 231

Malaysian Code on Take Over and Merger 231

\section{New Zealand}

Companies Act $1955 \quad 64$ s $34 \quad 59$

Sch 60

Third Sch, Table A, art $80 \quad \mathbf{6 4}$

Companies Act $1993 \mathbf{1 1 , 5 9 , 1 2 1 ,}$ $124,127-40,141,142,286$

s 2 127-8

s 128(1) 47

s 128(2) 47

s 128(3) 63

s $164 \quad 128$

s $174 \quad 128$

State Owned Enterprises Act 1986 s $4 \quad 129$

NZ Institute of Directors Code of Ethics $\mathbf{2 8 7}$
Securities Commission: Corporate Governance in NZ -

Principles and Guidelines, 2004 282, 283-4, 286, 288, 289, 290, 291

Pr 1283

Stock Exchange Listing Rules (2010) (NZSX) 19, 44, 127, 282, 284-6, 287

r $10.5 \quad \mathbf{2 8 7}$

r $10.5 .5 \quad 284$

r $10.5 .5(\mathrm{~h}) \quad \mathbf{2 8 5}, \mathbf{2 8 7}$

r 10.5.5(i) 285

App 16, Cl A 285

App 16 (Corporate Governance

Best Practice Code) 19,

284-5, 287, 288, 289, 290, 291

App 16, cl $1.2 \quad \mathbf{2 8 6}$

App 16, cl $1.3 \quad \mathbf{2 8 6}$

\section{Singapore}

Singapore Companies Act (SCA)

Pt VIIIA 238, 246

s $210 \quad 238$

Companies (Amendment) Act 1987246

\section{Taiwan}

Company Law 212, 224

Art $27 \quad 217$

Art $218 \quad 227$

Securities and Exchange Law, 2006213

Art 14-1 218

Art 14-2 228

Art 14-3 218

Art 36-1 218

Regulations Governing

Establishment of Internal

Control Systems by Public

Companies $\mathbf{2 2 0}$ 
United Kingdom

Bubble Act $1720 \quad \mathbf{5 1 , 5 2 , 5 3 , 2 7 7}$

Companies Act 1862 55, 56, 57, 61

s $14 \quad 60$

Table A $\mathbf{5 5 , 5 8 , 6 0}$

Table A, art 55 58-9, 65

Companies Act $1948 \quad \mathbf{6 4 ,} 234$

Companies Act $1985 \quad \mathbf{2 4 0 ,} 247$

ss $425-427 \quad \mathbf{2 4 0}$

s $425 \quad 240$

Table A, art $70 \quad 62$

Companies Act 2006 48, 56, 62,

$$
\text { 63, } 247
$$

s $18 \quad 48$

s $19 \quad \mathbf{4 8}$

s $1726,8,11,69,79,80,81$,

134

Companies Clauses Consolidation Act $1845 \quad \mathbf{5 5}$

s $90 \quad \mathbf{5 5}$

Enterprise Act (UK EA)

2002241

Pt $10 \quad 241$

Insolvency Act $1986 \mathbf{2 4 0}$

Pt II 238, 246

s $8 \quad 240$

Joint Stock Companies Act

$1844 \mathbf{8 , ~ 5 3 , ~ 5 4 , ~} 57$

s $27 \mathbf{5 5 , 6 0}$

Joint Stock Companies Act

$1856 \mathbf{9}, \mathbf{5 4}, \mathbf{5 5}, \mathbf{5 6}, \mathbf{5 7}, \mathbf{6 0}$, 61, 63

Table B 55, 58, 60

Table B, art $44 \quad \mathbf{6 5}$

Table B, art $46 \quad 58$

Limited Liability Act $1855 \quad \mathbf{5 4}$

Companies (Model Articles)

Regulations 2008, SI

2008/3229 62, 64
Combined Code: Financial

Reporting Council Combined

Code on Corporate

Governance, 200648

Charter of the Company of Clothworkers of London (1648) 59, 64-5

Charter of the Corporation of the Amicable Society for a Perpetual Assurance-Office (1710) 59, 65

Deed of Settlement of the Halifax Joint Stock

Banking Company, 183164

Deed of Settlement for the

London Ale Brewing

Company 64

\section{United States of America}

Alien Tort Claims Act $\mathbf{7 5 , 8 2}$

Bank Secrecy Act 185

Change in Bank Control Act, 2009 180, 200

Civil Monetary Penalty Inflation

Adjustment Rule 2008, corrected $2009 \mathbf{2 6 7}$

Clean Air Act 263, 267

Clean Water Act $\mathbf{2 6 7}$

Comprehensive Environmental

Response, Compensation

and Liability Act

(CERCLA) 255, 267

Debt Collections Improvement

Act 267

Dodd-Frank Wall Street Reform and Consumer Protection Act $2010 \quad \mathbf{2}, \mathbf{3}, \mathbf{7}, \mathbf{2 0}, 140$

s $951-7 \quad 2$

s 9712 
Emergency Planning and Community Right-to-Know Act $2006 \quad 267$

FDIC Improvement Act 1991 179, 200

Federal Aviation Administration Authorization Act (FAAA Act) $2006 \quad \mathbf{2 6 4}, 268$

Federal Civil Penalties Inflation

Adjustment Act, 1990 (2006) 267

Financial Institutions Reform, Recovery and Enforcement Act (FIRREA) $\mathbf{1 7 8}$

Glass-Steagall Act $\mathbf{1 6 8 ,}, 198$

Gramm-Leach-Bliley Act 1999198

Model Business Corporations Act (2003) 47, 62, 63, 198, 199, 200, 201

$$
\begin{aligned}
& \S 3.02(10) \quad \mathbf{2 0 1} \\
& \S 7.28 \quad \mathbf{1 9 9 ,} \mathbf{2 0 1} \\
& \S 15.05 \quad \mathbf{2 0 1}
\end{aligned}
$$

Public Accounting Reform and Investor Protector Act see Sarbanes-Oxley Act (SOX)

Resource Conservation and

Recovery Act (RCRA)

2006 255, 267

Sarbanes-Oxley Act 2002

(SOX) 15, 140, 191, 201, 212, 213, 280, 281

Securities Act 1933

$$
\text { s } 11 \quad \mathbf{1 8 6}, 187
$$

Securities Exchange Act 1934

s 10A 201

s 13(d) 157

s 14(a) 201

s 14(d) 201

s 14(e) 201

Toxic Substances Control Act $2006 \quad 267$
Code of Federal Regulations

(CFR)

12 CFR § 7.2014 (1996) 199

12 CFR § 7.5217(a) 199

17 CFR $\S \S 229.101$ (2010) 87

17 CFR $\S \S 229.103$ (2010) 87

17 CFR $\S \$ 229.303(2010) \quad 87$

17 CFR § 240 (1998) 260

US Code (USC)

12 USC $24 \quad 200$

12 USC $71 \quad 200$

12 USC $\S 1818(b)(3)$ (2008) 199, 200

12 USC 1818(e) 200

12 USC 1818(e)(1)(A)(ii)-(iii) (2008) 200

12 USC 1818(e)(1)(C)(i)-(ii) (2008) 200

12 USC § 1818(i) (2008) $\mathbf{2 0 0}$

12 USC $\S 1818(\mathrm{i})(2)(\mathrm{B})(\mathrm{i})$ (2008) 200

12 USC § 1818(i)(C) (2008) 200

12 USC $\S 1821(\mathrm{k}) \quad \mathbf{1 7 8}, \mathbf{1 7 9}$, 184, 188, 196, 200

12 USC $\S 1831$ o (2006) 199 12 USC § 1831 p-1 (2006) 199, 200

12 USC § 1842(a)(1)(2006) 199

12 USC § 1842(a)(2)(2006) 199

Restatement (Second) Conflict of Laws §302 (2009) 201

Delaware General Corporation Law (NB p 206, 2008, 2011) 47, 71, 133, 200

$\S 101(\mathrm{a}) \quad \mathbf{2 0 1}$

$\S 102(\mathrm{a})(2) \quad 201$

$\S 102(a)(4) \quad 198$ $\S 102$ (b)(7) 183, 184, 185, 188,

193, 194, 196

$\S 141 \quad 47$ 
$\S 153 \quad 198$

$\S 154 \quad 198$

$\S 170 \quad 198$

$\S 242(a)(3) \quad 198$

Georgia Business Corporation,

GA Code Ann tit 14, §14-2-

202(b)(5) 132

Federal (Corporate) Sentencing

Guidelines, $2010 \quad 281$

NASDAQ Listing Rules, 2009281

New York Stock Exchange Listing Rules (NTSE Rules 2010) 3, 281

Securities Exchange Commission (SEC)

r 14a 260

r $14 a-112$

Charter and Bylaws of the Great

Western Iron Company 1936

(State of Pennsylvania)

Art V 64

Maine Atlantic Granite Company, Bylaws (1836)

Art $2 \quad 59$

\section{European Union}

Registration, Evaluation,

Authorization and Restriction of Chemicals (REACH)
Regulation (EC) No

$1907 / 2006256$

\section{Conventions}

Framework Convention on

Climate Change $\mathbf{2 6 7}$

ILO Declaration on Fundamental

Principles and Rights at Work (ILO 1998) $\mathbf{7 6}$

International Covenant on

Civil and Political Rights

(ICCPR) 74, 75

International Covenant

on Economic, Social

and Cultural Rights

(ICESCR) $\mathbf{7 5}$

OECD Guidelines for

Multinational Enterprises (2008) 76

OECD Principles of Corporate

Governance 123

Rio Declaration on Environment and Development (1992) $\mathbf{7 6}$

UN Principles for Responsible Investment (UNPRI), 200584

Universal Declaration on Human

Rights 75

Art $30 \quad 76$

World Bank Principles, 2001

Principle $17 \quad 247$ 
P. M. Vasudev and Susan Watson - 9780857931535 Downloaded from PubFactory at 04/26/2023 10:48:26AM 